\title{
Pulmonary fibrosis and mineral dusts
}

\author{
J. C. WAGNER
}

From the Medical Research Council Pneumoconiosis Unit, Llandough Hospital, Penarth, S. Glamorgan, Wales

The reaction of the lungs to mineral dust depends on the dosage and the nature of the dust inhaled. The dusts to be considered are those which consist of particles of a suitable size to penetrate and be retained in the respiratory portions of the lungsthat is, beyond the terminal bronchioles. Spherical or irregular particles of less than $10 \mu \mathrm{m}$ in diameter and fibres of less than $3 \mu \mathrm{m}$ in diameter are of suitable size to penetrate to the lung parenchyma. A fibre is defined as a particle having a length/ diameter ratio of $3: 1$ or more. In fact, the fibres that are most commonly responsible for fibrosis of the lung have a ratio of $10: 1$ or greater. All dust particles less than these dimensions can penetrate to the respiratory airways. The lower limit of retention is more difficult to define. Particles of a dimension equivalent to a standard density sphere of less than $1 \mu \mathrm{m}$ in diameter have a low settling speed, and the smaller the particle the less likely it is to be deposited on the walls of the airways.

Dust particles that have been deposited on the walls of the peripheral airways (that is, below the terminal bronchioles, which are the smallest airways that have cilia and secrete mucus) may be removed by two alternative routes-either respiratory movement brings the particles up to the cilia in the terminal bronchioles, or they are carried by the lymph into the perivascular lymphatic channels. Most of the particles will have been phagocytosed by the alveolar macrophages, and the longer fibres may have several macrophages attached along their length.

By these mechanisms the lungs can reject small amounts of dusts. Fibrosis occurs only when these mechanisms become overloaded. This may be initiated by two alternative types of reaction, depending on the nature of the dust. In the first of these the dust accumulates in the alveoli arising directly from the respiratory bronchioles. These spaces become filled with macrophages containing dust and free lying particles, particularly if the dust is fibrous. Then a number of fibroblasts are attracted to the site. If the dust is relatively bland-for example, tin, iron, or pure carbon-the amount of fibrosis is slight and the macrophages and dust will be held in the alveoli by a thin web of reticulin fibres. If the dust is more cytotoxic the reaction will be more intense and dense collagen will obliterate the alveoli with extension into the supporting interstitium. This is the lesion caused by asbestos fibres. The second type is an interstitial reticuloendothelial reaction which is observed with quartz dust. The lesions in coal workers' pneumoconiosis are a mixture of these two responses. There is a marked difference in the amount of dust that is recovered from the lungs of cases of severe fibrosis in each of these conditions. According to Nagelschmidt $(1960,1965)$ the lungs of cases of severe PMF from South Wales contained $50 \mathrm{~g}$ of dust, and the lungs of a case of massive silicotic fibrosis in a Witwatersrand gold miner contained 4-10 $\mathrm{g}$ of dust, of which 1-3 g was quartz. The lungs of a severe case of asbestosis in a British factory worker contained $4 \mathrm{~g}$ of dust, of which the actual fibre weighed less than $1 \mathrm{~g}$ in both lungs. The effect on the macrophages of the various types of dust has already been described by Dr. A. C. Allison (p. 8).

\section{Asbestosis}

Asbestos is a term used to describe a number of fibrous silicates. Chrysotile, the commonest (95\%) of the exploited fibres, is a magnesium silicate the fibres of which when separated have a wavy, coiled appearance. The other two fibres to be considered are the non-silicate amphibole fibres, crocidolite and amosite, which between them account for about $4 \%$ of the commercially used asbestos.

When fibres are inhaled and retained in the lung parenchyma a number of them become coated with an iron-protein complex which is smooth, golden brown in colour and has bulbous ends (the socalled drumstick appearance). These bodies are striking when viewed under the light microscope, but electron microscopy studies reveal that these coated bodies are in the minority and are considerably outnumbered by the uncoated fibres, which may be so fine in diameter that there is insufficient resolution to see them without considerable magnification. They are more commonly formed around the amphibole fibres than the chrysotile (Pooley, 1972).

Lesions from the inhalation of asbestos dust consist initially of fibres, some in macrophages others lying free, asbestos bodies, and cell debris accumulating in the alveoli arising directly from the respiratory bronchioles. These deposits seem to become organized. Collagen fibres replace the thin reticulin web and spread into the wall of the 
respiratory bronchioles. At an early stage only a few scattered respiratory bronchioles are affected. As the disease progresses it affects more and more respiratory bronchioles, the fibrosis spreads upwards, into the walls of the terminal bronchioles, and peripherally into the whole of the acinus, eventually reaching the terminal airsacs. The individual lesions tend to spread and enmesh with each other, leading to a diffuse interstitial fibrosis. The intensity of the fibrous reaction increases if sufficient asbestos fibres have been retained until most of the lung parenchyma is replaced by a fibrous tissue network surrounding distorted, collapsed airspace and containing clumps of asbestos bodies and fibres.

The lesions in asbestosis first appear at the base of the lower lobes of the lungs, and the interstitial fibrosis gradually spreads upwards as the disease progresses. This progression may continue without further exposure to asbestos dust if sufficient fibre has been retained. All the commercial types of asbestos will cause pulmonary fibrosis if there has been sufficient exposure to respirable dust. Experiments with amosite dust have shown that fibres of less than $5 \mu \mathrm{m}$ in length do not cause fibrosis when inhaled by rats. Webster (1970) found only minimal reaction to crocidolite fibres less than $5 \mu \mathrm{m}$ in length in monkeys. There is no record of an inhalation experiment in which chrysotile fibres of this size were used.

The size range of fibres that can penetrate into the lung parenchyma in man has been shown by Timbrell (1970) to be less than $3 \mu \mathrm{m}$ in diameter, the length being variable. A few fine fibres of over $200 \mu \mathrm{m}$ in length can be inhaled into the respiratory airways if the individual is exposed to a large number of such fibres, most of which will not pass the nasal hairs. Fibres recovered from the human lung vary in size from more than $200 \mu \mathrm{m}$ to small particles that can be seen only under a transmission electron microscope. Fibres above $10 \mu \mathrm{m}$ in length are considered to be mainly responsible for pulmonary fibrosis. The malignant tumours associated with exposure to asbestos dust and asbestosis are not within the scope of this paper.

Fibrous asbestos dusts affect both the visceral and parietal pleura, and if the main reaction is from the visceral pleura there is a rapid increase of fibroblasts in the subpleural connective tissue usually associated with an increased vascularity. The vessels appear to become sclerosed and a dense fibrous tissue layer develops which can cover the whole lung and reach $1 \mathrm{~cm}$ in thickness. More commonly, fibres penetrate both visceral and parietal pleural layers and initiate a fibrous tissue reaction below the parietal mesothelial layer leading to the development of dense fibrous tissue with a typical basket-weave appearance (Meurman, 1966). In the centre of these lesions ischaemic necrosis occurs and calcium phosphate is gradually deposited leading, after many years, to large calcified pleural plaques. These plaques are seen usually in the lower thorax where they tend to follow the line of the ribs. Apart from the most common site, which is on the dome of the diaphragm, these plaques are occasionally seen on the visceral pleura as well as the parietal. Plaques rarely cause any disability. In some cases there may be large pleural plaques without parenchymal disease.

\section{Silicosis}

The interstitial reticuloendothelial (mononuclear/ macrophage) reaction to quartz takes place after the quartz crystals, usually in the $2-5 \mu \mathrm{m}$ range, have destroyed the macrophage, as described by Dr. Allison. The quartz crystals are then taken up by further macrophages and the process is repeated. If there is sufficient quartz present to overwhelm the macrophages the quartz is then taken up by the type II alveolar epithelial cells (granular pneumocytes). This results in these cells discharging their lamellar bodies and associated lipids from the lysosomes, leading to a localized alveolar proteinosis. If sufficient type II cells are involved a widespread alveolar proteinosis will occur.

Most of the fluid containing the active enzymes lipids, and quartz crystals is apparently removed by the lymphatics that initially drain the excess fluids from the lung parenchyma in channels which com municate with airways at the junction of the alveolar ducts and atria. At the level of the respiratory bronchioles there are usually four lymphatic channels surrounding the arterioles and others accompanying the marginal venules. Along the course of these lymphatics are small aggregations of lymphocytes, macrophage/mononuclear cells of the reticuloendothelial system, and occasional fibroblasts. Presumably the material from the airways is filtered through this clump of cells and the quartz crystals are taken up by the connective tissue histiocytes, which are destroyed in a similar manner to the alveolar phagocytes. The release of enzymes and the lipids encourages the production of reticulin fibres and more fibroblasts and macrophages are attracted to the area. This leads to the formation of a focus of perivascular dust reticulination in which the macrophages, fibroblasts, and other mononuclear cells become enmeshed in proliferating reticulin fibres surrounding the blood vessel. Many of the macrophages appear to have been destroyed and their content of quartz and other dust is lying free in the fibrous connective tissue. The fibroblasts produce collagen fibres which gradually replace the reticulin.

Some of the quartz and, presumably, the lipid and enzymes continue to progress along the lymphatics towards the hilum of the lung producing foci of dust 
reticulination where further aggregations of reticuloendothelial cells occur and, later, nodules in the lymph nodes. If there is sufficient exposure to quartz then the lymphatics leading to the visceral pleura become involved, leading to the development of fibrous nodules in the subpleural lymph nodes. At the same time that these lesions are proliferating the perivascular fibrous tissue is increasing concentrically around some of the blood vessels. The lymphatics are obliterated and the lumen of the blood vessels gradually become constricted until all that is left is the outline of the elastic layers which can be picked out if specific staining is used, in a mass of concentric collagen fibres. In some cases ischaemic necrosis occurs and the centre of the nodule will consist of greyish necrotic material.

Previously it was considered that the silicotic islet with a necrotic centre was caused by tuberculous infection, and these nodules were known as tuberculosilicotic islets. This possibly occurs in some cases with the larger foci but not in those of less than $2 \mathrm{~mm}$ in diameter, when it appears to be central ischaemic necrosis. As the collagen increases the centre of the nodules become acellular and most of the quartz tends to appear on the periphery, where there is a rim of dust reticulination in which macrophages are being destroyed. The fully matured silicotic islet consists of whorled concentric hyaline fibres. This glassy-like appearance of the collagen fibres is due to a coating consisting of degenerating blood proteins, mainly gamma-globulins.

In acute silicosis an overwhelming amount of quartz is rapidly deposited in the lung parenchyma. The centrilobular lymphatics appear to become completely blocked, and the fibrotic response extends along the perivenous lymphatics in the intralobular system leading to a perilobular interstitial fibrosis consisting of small fibrotic nodules. Foci of dust reticulination form along the course of the vein. In addition, there is proliferation and destruction of the type II alveolar epithelial cells resulting in either focal or generalized alveolar lipoproteinosis.

In workers who have had a moderate but prolonged exposure to quartz dust nodules may be found in lymph nodes beyond the hilum of the lung. The cervical lymph nodes are often affected and the paraaortic lymph nodes down to the level of the inguinal glands may also be affected.

The silicotic islets occur in the apical segments of the upper and lower lobes and are initially discrete but later coalesce to form conglomerate nodules. This fusion may become sufficiently extensive to cause large areas of massive conglomerate fibrosis. In a similar manner to asbestosis, the silicia lesions may progress without further exposure once a sufficient dosage of quartz has occurred. At any stage the whole histological appearance can be radically altered by tuberculosis. Cavitation in the massive lesions is usually associated with tuberculosis, but can occur as a sequel to ischaemia. Calcification commonly occurs after caseation or ischaemic necrosis. Peripheral calcification is commonly seen in the lymph nodes at the hilum of the lungs.

\section{Coal workers' pneumoconiosis}

Coal miners are exposed to a mixed dust consisting of coal, kaolin, mica, and silica. When this is inhaled and deposited beyond the terminal bronchioles it elicits a macrophage response. These macrophages phagocytose the particles and initially they are removed by the normal clearance mechanism through the cilia and mucus in the terminal bronchioles. If this route of clearance is not effective macrophages begin to accumulate in the alveoli which arise directly from the respiratory bronchioles. This occurs in people who are not miners living in areas polluted by the burning of coal, in whom a small amount of deeply pigmented dust can be seen in a centrilobular distribution.

In coal miners and those men who have worked in ships as coal trimmers (Gough, 1940) deposition is particularly seen in the alveoli of the second order of respiratory bronchioles (Heppleston, 1953). In some manner fibroblasts are attracted to the site and secrete a thin reticulin network which entraps the macrophages. Some of these lyse and more fibroblasts are attracted which produce more reticulin and the fibrotic process spreads to the walls of the respiratory bronchioles.

In some cases, particularly when the dust contains a small amount of quartz, collagen fibres are produced which coarsen the network. In this manner the three or four respiratory bronchioles arising from the terminal bronchiole may all have thickened, splinted walls which centrally come into apposition and in cross-section give the appearance of a centrilobular stellate nodule (Heppleston, 1953). In some cases the walls of these respiratory bronchioles become grossly thickened and give the impression of a focal intralobular interstitial fibrosis. In some cases these foci fuse with those of the adjoining lobules to produce a more extensive interstitial fibrosis. This interstitial fibrosis may be the result of repeated respiratory infections treated by antibiotics (Heppleston, 1972) or, as has been shown in a few cases, may be due to pigeon fancier's disease. Pigeon keeping is a common hobby among coal miners. (The subject of this paper is fibrosis, and the complex problems of emphysema and coal workers' pneumoconiosis are not discussed).

In some cases, possibly owing to the presence of quartz in the dust, the more significant lesion 
develops in a similar manner to that described in silicosis leading to a fibrotic nodule developing around the centrilobular arteriole. This nodule is not as circumscribed as that seen in silicosis. It usually consists of a mass of macrophages containing black dust, mononuclear cells, and a few fibroblasts surrounding the blood vessel, all held in place by a reticulin network. In most cases the lesions do not progress but in others the cellular component decreases and the fibres become more prominent with collagen fibres replacing the reticulin. This fibrous tissue encroaches on the lumen of the arteriole and in some lesions a network of capillaries appear. In others the central vessel is either completely sclerosed or there is an area of central ischaemic necrosis. These necrotic foci were described previously as 'infective nodules' suggesting tuberculosis, but, as in silicosis, this can be focal ischaemic necrosis.

The development of these nodules may be exacerbated by the presence of tubercle bacilli or rheumatoid factor. Depending upon the amount of dust in the lung and other features which are not understood, these nodules may remain small and discrete or enlarge and merge with others eventually to form the large, massive lesion of progressive massive fibrosis. In all these changes there is a possibility of vascular obliteration and foci of ischaemic necrosis are common. In some cases the greater part of the massive lesion may necrose leaving a thin capsule of fibrous tissue surrounding black fluid containing necrotic material.

The composition of these massive lesions in coal workers' pneumoconiosis has never been elucidated. Wagner et al. (1975) examined a small series of cases, studying biochemical, pathological, ultrastructural, and immunological features of the same material. The preliminary results suggest that collagen is present in the capsule of these lesions but that at the centre it is replaced by another insoluble protein (or proteins) which is probably stabilized by some form of cross-linking. This protein complex accounts for one-third of the weight of the lesions, the remaining two-thirds consisting of about equal amounts of mineral dusts and calcium phosphate. Serum proteins were also observed but their association with the lesions has yet to be determined.

Caplan (1953) first described a peculiar form of progressive massive fibrosis which occurred in coal miners with evidence of rheumatoid arthritis or who subsequently, in some cases years later, showed the features of the rheumatoid diathesis. The pathological appearance of the lesions were described by Gough et al. (1955). Rheumatoid pneumoconiosis has a consistent macroscopic and microscopic appearance, although occurring in nodules of various sizes. It used to be mistaken for tuberculosis or massive silicosis, both of which it may resemble, but the lesions contain minimal quartz and show no evidence of tuberculosis on bacterial investigation. Macroscopically the lesions appear as discrete nodules and also as masses built up of conglomerate nodules. Individual nodules are usually about $1.5 \mathrm{~cm}$ in diameter. Their cut surface shows concentric layers made up of black circles containing coal dust alternating with yellow or grey circles of necrotic tissue and fibrous material. These features may also be seen in the massive form, the individual concentric nodules still being identifiable. The amount of dust in the lung may be slight, or in other cases rheumatoid lesions are seen associated with the stellate lesion and nodules of coal workers' pneumoconiosis.

Gough et al. (1955) were impressed by the histological appearance of these lesions when is stained with haematoxylin and eosin. The centre $\frac{5}{0}$ of the nodule is necrotic and acellular, staining pink, then comes alternate layers of black coal dust and $N$ necrotic tissue. Outside this there is a blue-staining $\stackrel{\vec{N}}{\mathrm{~N}}$ zone of cellular infiltration where the macrophages, 은 polymorphonuclear leucocytes, and occasional giant cells appear to be actively destroying the collagen either in a complete circle around the lesion or in a segment. Around this zone pallisading cells are seen but far fewer than are seen in subcutaneous rheumatoid nodules. Cavities often occur. During $\overrightarrow{0}$ the inflammatory activity macrophages, whice $\searrow$ already contain dust, migrate to the lesion an when these die the dust is deposited. This accounts for concentric rings which develop and indicate periods of former activity. Outside this inflammatory zone is collagen in various stages of maturity. In the outer layer clumps of plasma cells and lymphocytes are seen. There is well marked endarteritis at the periphery of the lesions. This is distinguished from the endarteritic changes seen on the periphery of ordinary massive lesions by the presence of plasma cells within the obliterated lumen.

When the active inflammation subsides it is difficult to differentiate between a 'burnt out' 8 rheumatoid nodule, an old tuberculous focus, or an old silicotic nodule, since all these lesions may contain foci of calcification. However, many concentric rings of coal dust are seen most often in the rheumatoid lesions.

Wagner and McCormick (1967) reported on serological and immunofluorescent studies in cases of rheumatoid pneumoconiosis and ordinary coal $\mathrm{\omega}$ workers' pneumoconiosis. In the serological investigations sera from 450 men consisting of non- $c$ mining controls and four grades of pneumoconiosis (classified by radiography) and from patients with rheumatoid pneumoconiosis, in groups of $80-100$, were titrated against heat-aggregated human and rabbit gamma-globulin absorbed on tanned sheep 
red blood cells. It was notable that the incidence of positive results and the range of titres corresponded closely with the radiological order of progression of pneumoconiosis. On the whole, there were fewer positive tests with rabbit gamma-globulin as reactant and titres were lower with human gammaglobulin. In both test systems, however, there was a clear distribution of the highest titres in the Caplan cases. There is also an apparent anomaly in the Caplan cases where $26 \%$ were negative at all titres with human reactant and $43 \%$ with rabbit reactant, while most of the remainder of tests were positive at a titre of $1 / 640$ or above in both systems. Against the aggregated human gamma-globulin $74 \%$ of the Caplan cases, $30-40 \%$ of those with PMF, and only $2 \%$ of the controls had a titre of $1 / 160$ or greater.

Fresh tissue was obtained from all miners from one locality on whom a necropsy was performed and supplemented by cases from a chest hospital.
This material covered all categories of coal workers' pneumoconiosis. Fluorescein-labelled aggregated human gamma-globulin was used to detect rheumatoid factor. The cases were classified on histological criteria. Eight out of 10 cases showing the features of rheumatoid pneumoconiosis according to Gough et al. (1955) were positive. The second group to have a high rate of positivity, 10 out of 15 , were those cases of PMF with marked vasculitis in which plasma cell infiltration of the vessel walls extended into the obliterated lumina. These lesions were surrounded by numerous discrete foci of lymphocytes and plasma cells. In contrast, in the group classified as non-specific PMF only 3 out of 16 were positive. The possibility that these cases of PMF with vasculitis are an intermediate group, in whom the presence of rheumatoid factor is localized to the lung and hilar lymph glands and can be identified as such, requires fuller investigation. 